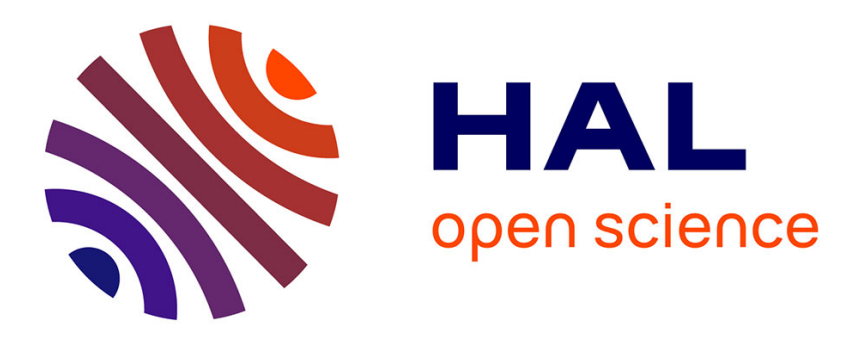

\title{
Discrete port-controlled Hamiltonian dynamics and average passivation
}

\author{
Alessio Moreschini, Mattia Mattioni, Salvatore Monaco, Dorothée \\ Normand-Cyrot
}

\section{- To cite this version:}

Alessio Moreschini, Mattia Mattioni, Salvatore Monaco, Dorothée Normand-Cyrot. Discrete portcontrolled Hamiltonian dynamics and average passivation. CDC 2019 - 58th IEEE Conference on Decision and Control, IEEE, Dec 2019, Nice, France. 10.1109/CDC40024.2019.9029809 . hal-02350436

\section{HAL Id: hal-02350436 \\ https://hal.science/hal-02350436}

Submitted on 6 Nov 2019

HAL is a multi-disciplinary open access archive for the deposit and dissemination of scientific research documents, whether they are published or not. The documents may come from teaching and research institutions in France or abroad, or from public or private research centers.
L'archive ouverte pluridisciplinaire HAL, est destinée au dépôt et à la diffusion de documents scientifiques de niveau recherche, publiés ou non, émanant des établissements d'enseignement et de recherche français ou étrangers, des laboratoires publics ou privés. 


\title{
Discrete port-controlled Hamiltonian dynamics and average passivation
}

\author{
Alessio Moreschini ${ }^{1,2}$, Mattia Mattioni ${ }^{1}$, Salvatore Monaco $^{1}$ and Dorothée Normand-Cyrot ${ }^{2}$
}

\begin{abstract}
The paper discusses the modeling and control of port-controlled Hamiltonian dynamics in a pure discrete-time domain. The main result stands in a novel differential-difference representation of discrete port-controlled Hamiltonian systems using the discrete gradient. In these terms, a passive output map is exhibited as well as a passivity based damping controller underlying the natural involvement of discrete-time average passivity.
\end{abstract}

Index Terms-Lyapunov methods; Energy systems; Algebraic/geometric methods

\section{INTRODUCTION}

Numerous physical systems as electromechanical and electrical systems admit a geometric structure, called portcontrolled Hamiltonian ( $\mathrm{pcH})$. These Hamiltonian dynamics are described in terms of a power-conserving geometric structure capturing the basic interconnections between different components of the process, and a Hamiltonian function given by the total stored energy. A major efficiency of these forms relies on the possibility to assign under feedback a desired energy, dissipation and equilibrium so referring to the well known Energy Shaping (ES) and Damping Injection (DI) or Interconnection and Damping Assignment (IDA) passivity based control which are powerful control strategies managing the energy balance between the different components of the process (see [1], [2]).

What has been said essentially refers to continuous-time modeling while a few recent work attack the discrete-time framework in terms of discrete gradient operators which were essentially developed in the literature to cope numerical integration aspects and variational calculus (see [3], [4]). In these work (see [5]-[8]), the discrete gradient operator is suitably used to capture energy exchanges and characterize power conservative or dissipative Hamiltonian structures in discrete time. However, many of these approaches rely on a given continuous-time $\mathrm{pcH}$ structure so giving rise under sampling to discrete structures which preserve conservative or dissipative properties in an approximate meaning which deserves some more specification (see [7]-[12]).

In this paper, discrete-time $\mathrm{pcH}$ dynamics are introduced in the difference/differential representation (DDR) context

Supported by Università degli Studi di Roma La Sapienza (Progetti di Ateneo 2018-Piccoli progetti RP11816436325B63) and by Université Franco-Italienne/Università Italo-Francese (Vinci Grants 2018 and 2019).

${ }^{1}$ Dipartimento di Ingegneria Informatica, Automatica e Gestionale A. Ruberti (La Sapienza University of Rome); Via Ariosto 25, 00185 Rome, Italy \{alessio.moreschini, mattia.mattioni, salvatore.monaco\}euniromal.it.

${ }^{2}$ Laboratoire des Signaux et Systèmes (L2S, CNRS); 3, Rue Joliot Curie, 91192, Gif-sur-Yvette, France \{dorothée.normand-cyrot, alessio.moreschini, \}el2s.centralesupelec.fr proposed in [13]. Exploiting such representation it is possible to split the dynamics into its free evolution expressed in terms of a discrete gradient with the desired interconnection and damping structural properties between the components, plus an input source. On these bases, an energy-balance equation is given so characterizing the relation between the stored, dissipated, and energy supplied by the source. In this scenario, average passivity as proposed in [14] naturally arises to define a passivating output. Its description in terms of the discrete gradient is discussed as well as the relative damping feedback strategies.

An interesting discussion is also performed to compare the proposed $\mathrm{pcH}$ structure with the one more commonly adopted in the literature which does not explicitly split the dynamics into its free and controlled evolutions but privileges the gradient form to more easily cope with the power conserving or dissipating properties of the dynamics. It is shown that these $\mathrm{pcH}$ forms and their associated passivating output can be reinterpreted in terms of average passivity from/around a certain control value [15]. Finally, the $\mathrm{pcH}$ models and control strategies here proposed are detailed in the constructive case represented by input-affine dynamics with quadratic Hamiltonian. In that case some optimality criteria can also be fulfilled. An academic example is discussed to illustrate the performances of the average passivity based stabilizing controller in a comparative way with respect to a more classical approach [7], [8]. Relations with more classical $\mathrm{pcH}$ forms in discrete time are discussed and the impact of the energy balance equation, which splits the supplied from the naturally dissipated energy (independently of the control) is clearly illustrated with a significant improvement of the damping performances with respect to the more usual passivity-based control strategies.

The paper is organized as follows. Preliminaries on discrete-time systems and discrete gradients are given in Section II. Discrete-time port-controlled Hamiltonian dynamics are introduced in Section III by enforcing energy properties in terms of $u$-average passivity. In Section IV, a further discussion with respect to classical discrete $\mathrm{pcH}$ modeling in the literature is performed by fully characterizing the dissipating energy. An example is discussed in Section $\mathrm{V}$ to illustrate the stabilizing performances of average passivity based damping controllers in a comparative spirit with more classical strategies. Concluding remarks are in Section VI.

\section{Preliminaries}

$\mathbb{R}$ and $\mathbb{N}$ denote, respectively, the set of real and natural numbers including 0 . For any vector $v \in \mathbb{R}^{n},|v|$ and $v^{\top}$ 
define the norm and transpose of $v$ respectively. Given a square matrix $R \geq 0$ and $v \in \mathbb{R}^{n}$, the weighted square seminorm is defined as $\|v\|_{R}^{2}:=v^{\top} R v$. For $v, w \in \mathbb{R}^{n}$, $\langle v, w\rangle$ denotes the inner product, i.e. $\langle v, w\rangle=v^{\top} w . I_{d}$ denotes the identity function or identity matrix while $I$ denotes the identity operator. Given a real-valued function $V(\cdot): \mathbb{R}^{n} \rightarrow \mathbb{R}$ assumed differentiable, $\nabla V$ represents its gradient vector when $\nabla$ denotes the $\mathbb{R}^{n}$ vector of partial derivatives. Given a smooth vector field over $\mathbb{R}^{n}$, the Lie operator is defined as $\mathrm{L}_{f}=\sum_{i=1}^{n} f_{i}(x) \frac{\partial}{\partial x_{i}}$. The arguments of the functions are dropped when clear from the context.

\section{A. Differential/Difference Representation}

As discussed in [13], the following couple of equations describe a nonlinear discrete-time single-input dynamics $\Sigma_{d}$

$$
\begin{aligned}
x^{+} & =x+F_{0}(x) \\
\frac{\mathrm{d} x^{+}(u)}{\mathrm{d} u} & =G\left(x^{+}(u), u\right) \quad \text { with } \quad x^{+}(0)=x^{+}
\end{aligned}
$$

where: $F_{0}(\cdot)$ is a $\mathbb{R}^{n}$-valued smooth map and $G(\cdot, u)$ is a vector field on $\mathbb{R}^{n}$ assumed complete; $x^{+}(u)$ represents a generic curve in $\mathbb{R}^{n}$ parameterized by $u \in \mathbb{R}$. When the initial condition $x^{+}(0)$ is fixed according to (1a) with $x^{+}(0)=x^{+}=x+F_{0}(x)$, completeness of $G(\cdot, u)$ ensures integrability of (1b) so recovering the usual representation in the form of a map. Namely, for all $\left(k, x_{k}, u_{k}\right) \in \mathbb{N} \times$ $\mathbb{R}^{n} \times \mathbb{R}$, equations (1) describe the one-step ahead discretetime dynamics: integrating (1b) for $u \in\left[0, u_{k}\right.$ [ with initial condition $x^{+}(0)=x_{k}+F_{0}\left(x_{k}\right)$, one recovers

$$
x_{k+1}=F\left(x_{k}, u_{k}\right)=x_{k}+F_{0}\left(x_{k}\right)+\int_{0}^{u_{k}} G\left(x^{+}(v), v\right) \mathrm{d} v \text {. }
$$

Hence, $x^{+}\left(u_{k}\right):=x_{k+1}$ represents the one-step ahead evolution while $x^{+}(0)=x^{+}$defines the one-step ahead evolution under the drift $F(x, 0):=x+F_{0}(x)$. For the equivalence among (2) and (1) the interested reader is referred to [13]. From now on, to simplify the notations, when no confusion arises the time arguments are dropped out.

Given a smooth mapping $\lambda(\cdot): \mathbb{R}^{n} \rightarrow \mathbb{R}$, the one-step ahead evolution of $\lambda(\cdot)$ along (1) can be computed through an integral form as in (2); namely,

$$
\lambda\left(x^{+}(u)\right)=\lambda\left(x^{+}(0)\right)+\int_{0}^{u} \mathrm{~L}_{G(., v)} \lambda\left(x^{+}(v)\right) \mathrm{d} v .
$$

\section{B. Average passivity in discrete-time}

The concept of average passivity has been introduced in [16] to relax the necessity of a direct input/output link that is unavoidable in discrete time to invoke passivity. Denoting by $\Sigma_{d}(h)$ the discrete-time system composed with the dynamics (1) and an output map $h(\cdot, u): \mathbb{R}^{n} \times \mathbb{R} \rightarrow \mathbb{R}$, the following definition is recalled.

Definition 2.1 (u-average passivity): Given the dynamics $\Sigma_{d}(h)$, let for any pair $(x, u) \in \mathbb{R}^{n} \times \mathbb{R}$, the $u$-average output map be defined as

$$
h^{a v}(x, u):=\frac{1}{u} \int_{0}^{u} h\left(x^{+}(v), v\right) \mathrm{d} v
$$

with $h^{a v}(x, 0):=h\left(x^{+}(0), 0\right)$ and $x^{+}(0)=x+F_{0}(x)$. It is said that $\Sigma_{d}(h)$ is $u$-average passive if there exists a positive semi-definite function $S(\cdot): \mathbb{R}^{n} \rightarrow \mathbb{R}_{\geq 0}$ (the storage function) such that, for all $(x, u) \in \mathbb{R}^{n} \times \mathbb{R}$

$$
S\left(x^{+}(u)\right)-S(x) \leq \int_{0}^{u} h\left(x^{+}(v), v\right) \mathrm{d} v=u h^{a v}(x, u) .
$$

It is worth mentioning that $u$-average passivity with respect to $h(\cdot, u)$ is equivalent to usual passivity with respect to $h^{a v}(\cdot, u)$.

In [15], the notion of $u$-average passivity from some nominal control value $\bar{u} \in \mathbb{R}$ has been introduced to deal with incremental-like passivity properties in discrete time. The following definition is hence recalled.

Definition 2.2 (u-average passivity from $\bar{u}$ ): $\Sigma_{d}(h)$ is $u$ average passive from $\bar{u} \in \mathbb{R}$ if there exists a function $S(\cdot)$ : $\mathbb{R}^{n} \rightarrow \mathbb{R}_{\geq 0}$ (the storage function) such that, for all $(x, u) \in$ $\mathbb{R}^{n} \times \mathbb{R}$, one verifies the inequality

$$
\begin{aligned}
\Delta S(x) & =S\left(x^{+}(\bar{u})\right)-S(x)+\int_{\bar{u}}^{u} \mathrm{~L}_{G(\cdot, v)} S\left(x^{+}(v)\right) \mathrm{d} v \\
& \leq \int_{\bar{u}}^{u} h\left(x^{+}(v), v\right) \mathrm{d} v
\end{aligned}
$$

with $\Delta S(x):=S\left(x^{+}(u)\right)-S(x)$ being the one step-ahead increment.

$u$-average passivity from $\bar{u}$ can be understood as $u$-average passivity of the dynamics around a nominal $\bar{u}$; namely,

$$
\int_{\bar{u}}^{u} h\left(x^{+}(v), v\right) \mathrm{d} v=\int_{0}^{u-\bar{u}} h\left(x^{+}(\bar{u}+v), \bar{u}+v\right) \mathrm{d} v .
$$

Defining the associated $u$-average output map

$$
h_{\bar{u}}^{a v}(x, u)=\frac{1}{u-\bar{u}} \int_{\bar{u}}^{u} h\left(x^{+}(v), v\right) \mathrm{d} v
$$

the inequality (6) rewrites as $\Delta S(x) \leq(u-\bar{u}) h_{\bar{u}}^{a v}(x, u)$ so recovering, when $\bar{u}=0$, classical $u$-average passivity.

Remark 2.1: $u$-average passivity from $\bar{u}$ is strictly reminiscent of the notion of incremental passivity (see [17]). It defines incremental-like passivity of the overall system with respect to trajectories that are parameterized by different inputs $u$ rather than time. Moreover, contrarily to incremental passivity, $u$-average passivity from $\bar{u}$ is referred to the influence of the incremental-like input $\Delta u=u-\bar{u}$ over the same output trajectories.

The following notion is instrumental for asymptotic stabilization through average passive output damping [14].

Definition 2.3 (Zero-state detectability): Given $\Sigma_{d}(h)$ let $x^{*} \in \mathbb{R}^{n}$ be an equilibrium and $\mathcal{Z} \in \mathbb{R}^{n}$ be the largest invariant set contained in $\left\{x \in \mathbb{R}^{n}\right.$ s.t. $\left.h\left(x^{+}(0), 0\right)=0\right\}$. $\Sigma_{d}(h)$ is said zero-state detectable (ZSD) if $x=x^{*}$ is an asymptotically stable equilibrium conditionally to $\mathcal{Z}$.

\section{Discrete gradient}

The notion of discrete gradient has been extensively studied (see [3], [4], [18] and references therein) with insights on numerical issues and facilities for computing it. In what follows, the standard definition is considered. 
Definition 2.4: Given a $C^{r}(r \geq 2)$ real-valued function $H(\cdot): \mathbb{R}^{n} \rightarrow \mathbb{R}$, its discrete gradient is the vector-valued function $\bar{\nabla} H(v, w): \mathbb{R}^{n} \times \mathbb{R}^{n} \rightarrow \mathbb{R}^{n}$ defined as

$$
\bar{\nabla} H(v, w)=\left.\bar{\nabla} H\right|_{v} ^{w}=\left[\begin{array}{lll}
\left.\bar{\nabla} H\right|_{v_{1}} ^{w_{1}} & \ldots & \left.\bar{\nabla} H\right|_{v_{n}} ^{w_{n}}
\end{array}\right]^{\top}
$$

with

$$
\left.\bar{\nabla} H\right|_{v_{i}} ^{w_{i}}=\frac{1}{w_{i}-v_{i}} \int_{v_{i}}^{w_{i}} \frac{\partial H\left(v_{1}, \ldots, v_{i-1}, \xi, w_{i+1}, \ldots, w_{n}\right)}{\partial \xi} \mathrm{d} \xi
$$

so verifying

$$
H(w)-H(v)=\left.(w-v)^{\top} \bar{\nabla} H\right|_{v} ^{w}
$$

with $\bar{\nabla} H(v, v)=\nabla H(v)$.

Remark 2.2: When $H(v)=\frac{1}{2} v^{\top} P v$ with square matrix $P \in \mathbb{R}^{n} \times \mathbb{R}^{n}$, then for any pair $(v, w)$, one easily computes, $\bar{\nabla} H(v, w)=\frac{1}{2} P(v+w)$.

\section{DisCRETE-TIME PORT-CONTROLLED HAMILTONIAN}

Although in continuous time energy-based modeling and control is a well consolidated approach exploiting Hamiltonian structures, in discrete time, discrete Hamiltonians have been mainly involved for numerical facilities. More recently, the discrete gradient operator has been suitably used to capture energy exchanges in discrete-time so characterizing power conservative or dissipative Hamiltonian discrete-time structures which rely, in a sampling context, to suitable approximations [6]-[12]. Herinafter, a novel differentialdifference representation of discrete port-controlled Hamiltonian systems is proposed. To this end, we start from Hamiltonian gradient dynamics described in free evolution in the standard implicit form

$$
x^{+}=x+\left.[J(x)-R(x)] \bar{\nabla} H\right|_{x} ^{x^{+}}
$$

with $x \in \mathbb{R}^{n}$ and $n$-dimensional matrix functions $J(x)=$ $-J^{\top}(x)$ and $R(x)=R^{\top}(x) \geq 0$ catching, respectively, the (power-preserving) interconnection and dissipative structure of the dynamics. The function $H(\cdot): \mathbb{R}^{n} \rightarrow \mathbb{R}$ defines the socalled Hamiltonian which is assumed bounded from below and characterizes the energy of (8). Discrete-time Hamiltonian dynamics verify the required energy and structural properties as recalled below.

Proposition 3.1: Consider the Hamiltonian dynamics (8) with $J(x)=-J^{\top}(x)$ and $R(x)=R^{\top}(x) \geq 0$ and $H(\cdot)$ : $\mathbb{R}^{n} \rightarrow \mathbb{R}_{\geq 0}$ being of class $C^{r}(r \geq 2)$ and possessing an equilibrium at $x^{*} \in \mathbb{R}^{n}$. Then, the following holds:

P1. the system is $H(\cdot)$-dissipative that is

$$
H\left(x^{+}\right)-H(x)=-\left\|\left.\bar{\nabla} H\right|_{x} ^{x^{+}}\right\|_{R}^{2} \leq 0, \quad \forall x \in \mathbb{R}^{n} ;
$$

P2. when $R(x)=0$ the system is $H(\cdot)$-conservative, namely $H\left(x^{+}\right)-H(x)=0, \forall x \in \mathbb{R}^{n}$;

P3. if $H\left(x^{*}\right)=0$ and $\bar{\nabla} H\left(x^{*}, x^{*}\right)=\nabla H\left(x^{*}\right)=0, x^{*}$ is a stable equilibrium;

P4. if $H\left(x^{*}\right)=0$ and $\bar{\nabla} H\left(x^{*}, x^{*}\right)=\nabla H\left(x^{*}\right)=0, x^{*}$ is an asymptotically stable equilibrium if the largest invariant set for which $\left\|\bar{\nabla} H\left(x, x^{+}\right)\right\|_{R}^{2}=0$ is $\left\{x^{*}\right\}$.
Proof: By computing the forward difference of $H(\cdot)$ along (8), and exploiting that $\left\|\left.\bar{\nabla} H\right|_{x} ^{x^{+}}\right\|_{J}^{2}=0$, one gets $\Delta H(x)=-\left\|\left.\bar{\nabla} H\right|_{x} ^{x^{+}}\right\|_{R}^{2}$ so that $\mathbf{P 1}$ and $\mathbf{P 2}$ immediately follow. Moreover, $H$ possesses a (local) extremum at $x^{*}$ so that $\left.\bar{\nabla} H\right|_{x^{*}} ^{x^{*}}=\nabla H\left(x^{*}\right)=0$ that is stable if $H\left(x^{*}\right)=0$ and from P1 so that $\mathbf{P 3}$ follows. Finally, P4 follows from the Barbashin-Krasovskii theorem.

In (8), the discrete gradient dynamics is implicitly defined. It can be explicitly described through a suitably defined function $x+F_{0}(x)$ as in (1a). It might be tough to compute such form which directly depends on the nonlinearity of the Hamiltonian function except for some polynomial cases. As an example, in the quadratic case, $H(x)=\frac{1}{2} x^{\top} P x$ with $P=P^{\top}$, the dynamics (8) can be explicitly computed according to Remark 2.2 so getting

$$
\begin{aligned}
x^{+} & =x+F_{0}(x) \\
& =\left(I-\frac{1}{2}[J(x)-R(x)] P\right)^{-1}\left(I+\frac{1}{2}[J(x)-R(x)] P\right) x .
\end{aligned}
$$

\section{A. Discrete-Time pcH dynamics}

In the present Differential Difference framework discretetime port-controlled Hamiltonian dynamics are defined as follows

$$
\begin{aligned}
x^{+} & =x+\left.[J(x)-R(x)] \bar{\nabla} H\right|_{x} ^{x^{+}} \\
\frac{\mathrm{d} x^{+}(u)}{\mathrm{d} u} & =G\left(x^{+}(u), u\right) \quad \text { with } x^{+}(0)=x^{+}
\end{aligned}
$$

with $x \in \mathbb{R}^{n}$, free evolution (9b) as in (8) and controlled evolution as in (1b).

Equivalently, a discrete-time pcH dynamics in the form of a map can be deduced through integration of $(9 \mathrm{~b})$ between 0 and $u$ with initial condition $x^{+}(0)=x^{+}$so getting

$$
x^{+}(u)=x+\left.[J(x)-R(x)] \bar{\nabla} H\right|_{x} ^{x^{+}}+u g(x, u)
$$

where by definition $u g(x, u):=\int_{0}^{u} G\left(x^{+}(v), v\right) \mathrm{d} v$ defines the input source. The pcH dynamics (9) verifies well-defined energy properties in terms of $u$-average passivity in straight relation with the discrete gradient. As a matter of fact, introducing the output map

$$
h(\cdot, u)=G^{\top}(\cdot, u) \nabla H(\cdot)=\mathrm{L}_{G(\cdot, u)} H(\cdot)
$$

and exploiting the integral form (3) for computing the forward difference $\Delta H(x)=H\left(x^{+}(u)\right)-H(x)$ of the Hamiltonian along (9), one directly writes down the $u$ average energy balance equality between two time steps as

$$
\underbrace{\Delta H(x)}_{\text {stored energy }}=-\underbrace{\left\|\left.\bar{\nabla} H\right|_{x} ^{x^{+}}\right\|_{R}^{2}}_{\text {dissipated energy }}+\underbrace{\int_{0}^{u} h\left(x^{+}(v), v\right) \mathrm{d} v}_{\text {supplied energy }} .
$$

holding for all $(x, u) \in \mathbb{R}^{n} \times \mathbb{R}$.

With this in mind the following theorem can be proved.

Theorem 3.1: Given a discrete $\mathrm{pcH}$ dynamics (9) (or, equivalently, (10)) with output map $h(\cdot, u)$ defined in (11) and Hamiltonian $H(\cdot): \mathbb{R}^{n} \rightarrow \mathbb{R}_{\geq 0}$ with $H(x)>0$ for all $x \in \mathbb{R}^{n} \backslash\left\{x^{*}\right\}$, then the following holds: 
(i) the dynamics (9) with output (11) is $u$ average passive with dissipation rate $d(x):=$ $\left\|\bar{\nabla} H\left(x, x^{+}\right)\right\|_{R}^{2} \geq 0$

(ii) when $R(x) \equiv 0$, (9) is $u$-average lossless;

(iii) the average output associated to (11) according to definition (4) takes the form

$$
h^{a v}(x, u)=\left.g^{\top}(x, u) \bar{\nabla} H\right|_{x^{+}} ^{x^{+}(u)}
$$

(iv) the feedback $u=\gamma(x)$ solution to the implicit damping equality

$$
u+\left.\kappa g^{\top}(x, u) \bar{\nabla} H\right|_{x^{+}} ^{x^{+}(u)}=0, \quad \kappa>0
$$

makes the closed-loop equilibrium $x^{*}$ of (9) (or, equivalently, (10)) globally asymptotically stable provided that ZSD with respect to $h(\cdot, u)$ holds.

Proof: $(i)$ and $(i i)$ follow from the energy dissipation equality (12) that implies, as $R(\cdot) \geq 0$ and Proposition 3.1,

$$
H\left(x^{+}(u)\right)-H(x) \leq \int_{0}^{u} h\left(x^{+}(v), v\right) \mathrm{d} v=u h^{a v}(x, u)
$$

holding with a strict equality whenever $R(\cdot)=0$. Moreover, one has $\Delta H(x)=H\left(x^{+}(u)\right)-H\left(x^{+}\right)+H\left(x^{+}\right)-H(x)$. From Proposition 3.1, one has that $H\left(x^{+}\right)-H(x)=$ $-\left\|\bar{\nabla} H_{x}^{x^{+}}\right\|_{R}^{2}$ whereas, by Definition 2.4, $H\left(x^{+}(u)\right)-$ $H\left(x^{+}\right)=\left.\left(x^{+}(u)-x^{+}\right)^{\top} \bar{\nabla} H\right|_{x^{+}} ^{x^{+}(u)}$ with, because of (10), $x^{+}(u)-x^{+}=g(x, u) u$. Thus, one gets that $\Delta H(x)=$ $-\left\|\left.\bar{\nabla} H\right|_{x} ^{x^{+}}\right\|_{R}^{2}+\left.u g^{\top}(x, u) \bar{\nabla} H\right|_{x^{+}} ^{x^{+}(u)}$. By equating the righthand sides of the above equality and (12), one gets that the average output rewrites in terms of the discrete gradient as

$$
h^{a v}(x, u)=\frac{1}{u} \int_{0}^{u} h\left(x^{+}(v), v\right) \mathrm{d} v=\left.g^{\top}(x, u) \bar{\nabla} H\right|_{x^{+}} ^{x^{+}(u)}
$$

so that (iii) follows. From the energy-balance equation (12) and Proposition 3.1, because $x^{*}$ is a minimum of $H(\cdot)$, we have that it is also a stable equilibrium for (10) when $u=0$. As a consequence, all feedbacks making the righthand side of (12) negative semi-definite ensure globally asymptotic stability of the equilibrium provided ZSD holds. As a consequence, the feedback solution to the damping equality (14) makes $\left.\Delta H(x)\right|_{u=\gamma(x)} \leq 0$ for all $x \in \mathbb{R}^{n}$ so that $(i v)$ follows.

In Theorem 3.1, it is shown that, as usual in continuous time, pcH systems come with a straightforward energy interpretation and direct passivity property. However, those features naturally involve, in discrete time, $u$-average passivity arguments. Moreover, in this scenario, the $u$-average output (13) rewrites in terms of the discrete gradient evaluated along the controlled evolution of (10) in a way that is strictly reminiscent of the continuous-time counterpart.

Remark 3.1: Theorem 3.1 expresses that the energy is non-increasing (conservative) or decreasing (dissipative) up to an isolated minimum of $H(x)$ and the change of internal energy is governed by both the energy-storage and energydissipation. Accordingly, a passivity-based stabilizing controller can be deduced by solving (14).
Remark 3.2: The stabilizing damping feedback $u=\gamma(x)$ is the implicit solution to the equality (14) whose solvability is guaranteed by $u$-average passivity [16]. An exact solution cannot be computed in general albeit approximations have been proposed in [19], [20] by solving (14) around $u=0$ so getting $u^{*}=-\kappa \lambda(x) g^{\top}(x, 0) \nabla H\left(x+F_{0}(x)\right)$ with a suitably tuned gain $\lambda(\cdot)>0$. By passivation, those solutions preserve global asymptotic stability in closed loop.

Remark 3.3: The $u$-average output (13) associated to (11) recovers the discrete gradient of the energy $H(\cdot)$ defined with respect to $u$ differentiation instead of $x$ as in Definition 2.4; i.e. $h^{a v}(x, u)=\frac{1}{u} \int_{0}^{u} \frac{\mathrm{d} H\left(x^{+}(v)\right)}{\mathrm{d} v} \mathrm{~d} v=\left.\bar{\nabla}_{u} H\left(x^{+}(u)\right)\right|_{0} ^{u}$ with by defintion of $G(\cdot, v), \mathrm{d} x^{+}(v)=\frac{\mathrm{d} x^{+}(v)}{\mathrm{d} v} \mathrm{~d} v=$ $G\left(x^{+}(v), v\right) \mathrm{d} v$.

Remark 3.4: The differential-difference representation (9) of pcH dynamics easily extends to the multi-input case when setting $\underline{u}=\left(u_{1}, \cdots u_{m}\right)^{\top} \in \mathbb{R}^{m}$ and defining the $\mathbb{R}^{m}$ output vector $\underline{h}^{a v}(x, \underline{u})=\left(h_{1}^{a v}(x, \underline{u}), \cdots h_{m}^{a v}(x, \underline{u})\right)^{\top} \in \mathbb{R}^{m}$ with, for $i=1, \ldots, m, h_{i}^{a v}(x, \underline{u}):=\left.\bar{\nabla}_{u_{i}} H\left(x^{+}(\underline{u})\right)\right|_{0} ^{u_{i}}$.

\section{B. Input-affine dynamics with quadratic Hamiltonian}

In this section, we detail our results to the class of discrete $\mathrm{pcH}$ systems in input-affine form

$$
x^{+}(u)=x+\left.[J(x)-R(x)] \bar{\nabla} H\right|_{x} ^{x^{+}}+u g(x)
$$

with by definition $u g(x):=\int_{0}^{u} G\left(x^{+}(v), v\right) \mathrm{d} v$ and quadratic Hamiltonian function $H(x)=\frac{1}{2} x^{\top} P x$ with $P=P^{\top}>0$. We show that the feedback solution to (14), with a fixed gain $\kappa>0$, is also optimal with respect to the criterium

$$
I=\frac{1}{2} \sum_{i=0}^{\infty}\left(l\left(x_{i}\right)+\left\|u_{i}\right\|^{2}\right), \quad l(\cdot) \geq 0 .
$$

Proposition 3.2: Consider the pcH dynamics (15) with Hamiltonian $H(x)=\frac{1}{2} x^{\top} P x$ with $P>0$ and output map $h(x, u)=\nabla H(x) G(x, u)$ then, there exists a unique solution to the damping equality (14) which minimizes (16) with

$$
l(x):=2\left\|\bar{\nabla} H\left(x, x^{+}\right)\right\|_{R}^{2}+\frac{\left\|g^{\top}(x) P x^{+}\right\|^{2}}{1+g^{\top}(x) P g(x)} .
$$

Such a feedback is given by $u=\gamma(x)$ with

$$
\gamma(x)=-\frac{2 \kappa^{*} g^{\top}(x) P x^{+}}{2+\kappa^{*} g^{\top}(x) P g(x)}
$$

and $\kappa^{*}=\left(1+g^{\top}(x) \frac{P}{2} g(x)\right)^{-1}$.

Proof: The proof is constructive. The implicit form (8) explicitly rewrites as $x^{+}=x+F_{0}(x)=$ (I $\left.\frac{1}{2} A(x)\right)^{-1}\left(\mathrm{I}+\frac{1}{2} A(x)\right) x$ for $A(x)=(J(x)-R(x)) P$ so that $h^{a v}(x, u)=g^{\top}(x) \frac{P}{2}\left(u g(x)+2 x^{+}\right)$. Solving the damping equality (14) which returns $u=-\kappa h^{a v}(x, u)$ one gets $\gamma(x)=-\frac{2 \kappa g^{\top}(x) P x^{+}}{2+\kappa g^{\top}(x) P g(x)}$. On the other hand, by defining $\mathcal{H}(x, u)=\frac{l(x)}{2}+\frac{\|u\|^{2}}{2}+H\left(x^{+}(u)\right)-H(x)$ the optimal control for (16) with (17) is provided by $u^{*}=-\frac{g^{\top}(x) P x^{+}}{1+g^{\top}(x) P g(x)}$ as solution to $\left.\frac{\partial \mathcal{H}}{\partial u}\right|_{u^{*}}=0$. Therefore, $u^{*}=\gamma(x)$ in (18) with gain fixed according to $\kappa=\kappa^{*}=\left(1+g^{\top}(x) \frac{P}{2} g(x)\right)^{-1}$. 


\section{AN INSIGHT INTO THE DISSIPATING ENERGY}

The energy-balance (12) emphasizes that the total stored energy can be exactly split into the naturally dissipated and supplied components. This is not the case when considering the discrete $\mathrm{pcH}$ form more commonly adopted in the literature following approximate or sampling inspired approaches [6]-[8], [21]. Let us discuss more in detail this aspect which directly affects energy control design.

A more standard pcH-dynamics takes the form

$$
x^{+}(u)=x+\left.[J(x)-R(x)] \bar{\nabla} H\right|_{x} ^{x^{+}(u)}+u \tilde{g}(x, u)
$$

which could be generalized to

$$
x^{+}(u)=x+\left.[\mathcal{J}(x, u)-\mathcal{R}(x, u)] \bar{\nabla} H\right|_{x} ^{x^{+}(u)}+u \tilde{g}(x, u) .
$$

These forms essentially differ from (10) where the discrete gradient is used to represent the drift only. Note that, in general, the additional input channel $\tilde{g}(\cdot, u)$ is assumed not input-dependent as well as the damping and interconnection terms $(\tilde{g}(x, u)=\tilde{g}(x), \mathcal{J}(x, u)=J(x)$ and $\mathcal{R}(x, u)=$ $R(x)$ ). Using (19), the energy balance (12) rewrites as

$$
\underbrace{\Delta H(x)}_{\text {stored energy }}=-\underbrace{\left\|\left.\bar{\nabla} H\right|_{x} ^{x^{+}(u)}\right\|_{R}^{2}}_{\text {dissipation }}+\underbrace{\left.u \tilde{g}^{\top}(x, u) \bar{\nabla} H\right|_{x} ^{x^{+}(u)}}_{\text {partial supplied energy }}
$$

where by construction the dissipated energy includes internal dissipation (due to control-free dynamics) and an inputdependent component. It results that (21) deduced from (19) does not emphasize the total energy supplied by the external source and this enlightens a critical aspect when dealing with energy-inspired control strategies.

To point this aspect, let us look at the results of the passivity-based-like control actions deduced from the representations (19) and (10) respectively. Starting from (19), partial passivity with respect to the output $y_{\ell}(x, u)=$ $\left.\tilde{g}^{\top}(x, u) \bar{\nabla} H\right|_{x} ^{x^{+}(u)}$ is straightforward from (21). Setting $u_{\ell}=-\kappa y_{\ell}$ with $\kappa>0$, one gets in closed loop

$$
\begin{aligned}
x^{+}\left(u_{\ell}\right)= & x+[J(x)-R(x) \\
& \left.\left.-\kappa \tilde{g}\left(x, u_{\ell}\right) \tilde{g}^{\top}\left(x, u_{\ell}\right)\right)\right]\left.\bar{\nabla} H\right|_{x} ^{x^{+}\left(u_{\ell}\right)}
\end{aligned}
$$

so preserving the Hamiltonian structure. However, the resulting closed loop energy balance rewrites

$\left.\Delta H(x)\right|_{u_{\ell}}=-\left\|\left.\bar{\nabla} H\right|_{x} ^{x^{+}\left(u_{\ell}\right)}\right\|_{R}^{2}-\kappa\left\|\left.\tilde{g}^{\top}\left(x, u_{\ell}\right) \bar{\nabla} H\right|_{x} ^{x^{+}\left(u_{\ell}\right)}\right\|^{2}$

The effect of damping is not clearly specified because of the partial nature of passivity which makes reference to the inner product $u y_{\ell}$ which does not represent the total supplied energy. On the other hand, when referring to (10) and $u$-average passivity, the inner product $u h^{a v}(x, u)$ does represent the total supplied energy. Then, the corresponding damping feedback $u^{a v}=-\kappa h^{a v}\left(x, u^{a v}\right)$ provides

$$
\left.\Delta H(x)\right|_{u^{a v}}=-\left\|\left.\bar{\nabla} H\right|_{x} ^{x^{+}}\right\|_{R}^{2}-\kappa\left\|\left.g^{\top}\left(x, u^{a v}\right) \bar{\nabla} H\right|_{x^{+}} ^{x^{+}\left(u^{a v}\right)}\right\|^{2}
$$

which explicitly specifies the effect of damping. The feedback system (10) takes the form

$$
\begin{aligned}
x^{+}\left(u^{a v}\right)= & x+\left.[J(x)-R(x)] \bar{\nabla} H\right|_{x} ^{x^{+}} \\
& -\left.\kappa g\left(x, u^{a v}\right) g^{\top}\left(x, u^{a v}\right) \bar{\nabla} H\right|_{x^{+}} ^{x^{+}\left(u^{a v}\right)} .
\end{aligned}
$$

so emphasizing two discrete gradient parts with respect to the free and controlled evolutions respectively.

Under which conditions are the advantages of both representations preserved in terms of energy split and preservation of the structure under feedback? It is a matter of computations to verify that both representations are equivalent when

$u g(x, u)=[J(x)-R(x)]\left(\left.\bar{\nabla} H\right|_{x} ^{x^{+}(u)}-\left.\bar{\nabla} H\right|_{x} ^{x^{+}}\right)+u \tilde{g}(x, u)$

with $x^{+}(u)=x+\left.[J(x)-R(x)] \bar{\nabla} H\right|_{x} ^{x^{+}}+u g(x, u)$, so clearly linking the structures of $g(\cdot, u)$ and $\tilde{g}(\cdot, u)$. The following result holds.

Proposition 4.1: Consider the pcH dynamics (10) with Hamiltonian $H(\cdot): \mathbb{R}^{n} \rightarrow \mathbb{R}_{\geq 0}$ and assume the existence of $\bar{u}$ as the solution to

$$
\begin{aligned}
& \left.(J(x)-R(x)) \bar{\nabla} H\right|_{x} ^{x^{+}}+g(x, \bar{u}) \bar{u} \\
= & \left.(\mathcal{J}(x, \bar{u})-\mathcal{R}(x, \bar{u})) \bar{\nabla} H\right|_{x} ^{x^{+}(\bar{u})}
\end{aligned}
$$

with $x^{+}(\bar{u})=x+\left.[J(x)-R(x)] \bar{\nabla} H\right|_{x} ^{x^{+}}+\bar{u} g(x, \bar{u})$ and properly defined matrices $\mathcal{J}(\cdot, u)=-\mathcal{J}^{\top}(\cdot, u), \mathcal{R}(\cdot, u)=$ $\mathcal{R}^{\top}(\cdot, u) \geq 0$; then, the following holds:

(i) $\mathcal{J}(\cdot, u), \mathcal{R}(\cdot, u)$ solve the matching equation

$$
\begin{aligned}
& g^{\perp}(x, u)\left[\left.(\mathcal{J}(x, u)-\mathcal{R}(x, u)) \bar{\nabla} H\right|_{x} ^{x^{+}(u)}\right. \\
& \left.-\left.(J(x)-R(x)) \bar{\nabla} H\right|_{x} ^{x^{+}}\right]=0
\end{aligned}
$$

with $g^{\perp}(\cdot, u)$ such that $g^{\perp}(\cdot, u) g(\cdot, u)=0$;

(ii) the dynamics (10) with output (11) is $u$-average passive from $\bar{u}$.

Proof: $(i)$ immediately follows from the definition of $\bar{u}$. For proving $(i i)$, exploiting the differential-difference form and $u$-average passivity, the one step ahead increment splits as

$$
\begin{aligned}
& \Delta H(x)=H\left(x^{+}\right)-H(x)+\int_{0}^{u} h\left(x^{+}(v), v\right) \mathrm{d} v= \\
& H\left(x^{+}\right)-H(x)+\int_{0}^{\bar{u}} h\left(x^{+}(v), v\right) \mathrm{d} v+\int_{\bar{u}}^{u} h\left(x^{+}(v), v\right) \mathrm{d} v .
\end{aligned}
$$

By definition of $\bar{u}$ as solution to (22), one gets

$H\left(x^{+}(\bar{u})\right)-H(x)=H\left(x^{+}\right)-H(x)+\int_{0}^{\bar{u}} h\left(x^{+}(v), v\right) \mathrm{d} v$ satisfying $H\left(x^{+}(\bar{u})\right)-H(x)=-\left\|\left.\bar{\nabla} H\right|_{x} ^{x^{+}(\bar{u})}\right\|_{\mathcal{R}}^{2}$. One gets $H\left(x^{+}(u)\right)-H(x) \leq \int_{\bar{u}}^{u} h\left(x^{+}(v), v\right) \mathrm{d} v$ so proving $(i i)$.

The above proposition allows to explicit the part of the supplied energy that is structurally dissipated by the system. Also, passivity can be deduced in a very natural way by recurring to $u$-average passivity around $\bar{u}$ solution to (22). Such a nominal dummy input is intuitively revealing the component of the source $u$ (and thus the one of (9)) whose energy is intrinsically dissipated by the dynamics. 

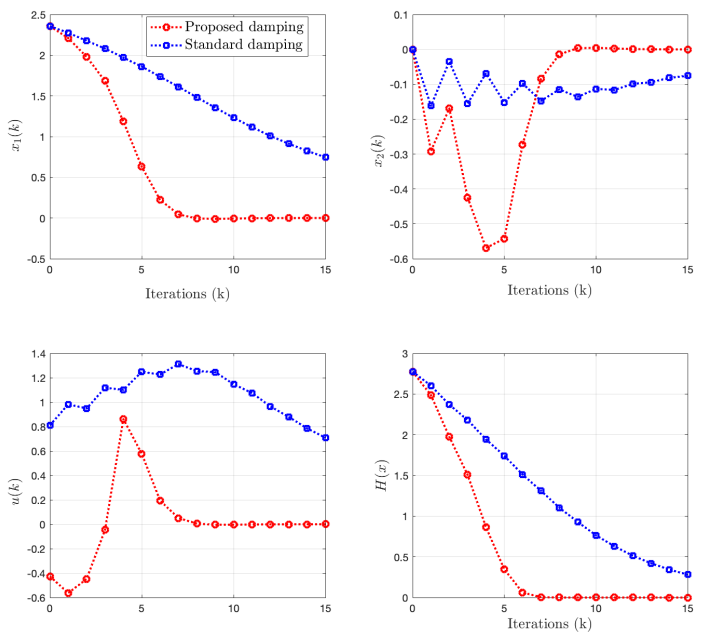

Fig. 1. Simulations for $x_{0}=\operatorname{col}\left(\frac{3 \pi}{4}, 0\right)$

\section{AN ACADEMIC EXAMPLE}

We consider the pcH dynamics

$$
x^{+}(u)=x+\frac{1}{2}\left(J-R\left(x_{1}\right)\right)\left(x^{+}(u)+x\right)+B u
$$

with $H(x)=\frac{1}{2} x^{\top} x, x=\operatorname{col}\left(x_{1}, x_{2}\right)$ and, for $\mu>0$

$$
J=\left(\begin{array}{cc}
0 & 1 \\
-1 & 0
\end{array}\right), R\left(x_{1}\right)=\left(\begin{array}{cc}
0 & 0 \\
0 & \mu\left(1-x_{1}^{2}\right)^{2}
\end{array}\right), B=\left(\begin{array}{l}
0 \\
1
\end{array}\right) .
$$

It is a matter of computation to verify that (24) is equivalent to the form (10) as

$$
x^{+}(u)=x+\frac{1}{2}\left(J-R\left(x_{1}\right)\right)\left(x^{+}+x\right)+g\left(x_{1}\right) u
$$

with $x^{+}=\left(I-A\left(x_{1}\right)\right)^{-1}\left(I+A\left(x_{1}\right)\right) x, g\left(x_{1}\right)=(I-$ $\left.A\left(x_{1}\right)\right)^{-1} B$ and $A\left(x_{1}\right)=\frac{1}{2}\left(J-R\left(x_{1}\right)\right)$. As a consequence of Theorem 3.1, (24) is $u$-average passive with $u$-average output (13) $h^{a v}(x, u)=g^{\top}\left(x_{1}\right)\left(x^{+}+\frac{1}{2} g\left(x_{1}\right) u\right)$ with the stabilizing feedback solution to (14) of the form

$$
u=-\frac{\kappa g^{\top}\left(x_{1}\right)}{1+\frac{\kappa}{2} g^{\top}\left(x_{1}\right) g\left(x_{1}\right)} x^{+}, \kappa>0
$$

globally asymptotically stabilizing the origin of (24).

Remark 5.1: The feedback discussed in Section IV is given by $u_{\ell}(x)=-\frac{\kappa B^{\top}\left(I-A\left(x_{1}\right)\right)^{-1}}{1+\frac{\kappa}{2} B^{\top} g\left(x_{1}\right)} x$ computed from the output $y_{\ell}(x, u)=\frac{1}{2} B^{\top}\left(x^{+}(u)+x\right)$ and $\kappa \geq 0$.

Figure 1 details the simulation results of the $u$-average passivity-based feedback (26) computed over (24). The results are compared to the feedback reported in Remark 5.1 for the same gain $\kappa=10$. The initial conditions are set to $x_{0}=\operatorname{col}\left(\frac{3 \pi}{4}, 0\right)$ while $\mu=0.8$. Simulations reveal that the proposed stabilizing feedback is indeed stabilizing the origin with smooth trajectories and limited control effort while guaranteeing a non-increasing Hamiltonian. In addition, it shows that the new feedback proposed in Theorem 3.1 is positively comparable the standard passivity-based feedback in [7], [8] for pcH dynamics even under different outputs.

\section{CONCLUSIONS AND FUTURE PERSPECTIVES}

A new formalization for $\mathrm{pcH}$ dynamics is introduced in terms of discrete gradient. Damping controllers are thus described making reference to a $u$-average passivating output. Further works concern the development of new energy shaping and damping assignment strategies [7], [11], [12] and the preservation of these forms under sampling.

\section{REFERENCES}

[1] A. J. van Der Schaft, L2-gain and passivity techniques in nonlinear control. Springer, 2000, vol. 2.

[2] R. Ortega, A. Van Der Schaft, B. Maschke, and G. Escobar, "Interconnection and damping assignment passivity-based control of portcontrolled Hamiltonian systems," Automatica, vol. 38, no. 4, pp. 585596, 2002.

[3] R. I. McLachlan, G. Quispel, and N. Robidoux, "Geometric integration using discrete gradients," Philosophical Transactions of the Royal Society of London A: Mathematical, Physical and Engineering Sciences, vol. 357 , no. 1754 , pp. 1021-1045, 1999.

[4] O. Gonzalez, "Time integration and discrete Hamiltonian systems," Journal of Nonlinear Science, vol. 6, no. 5, p. 449, 1996.

[5] A. Moreschini, S. Monaco, and D. Normand-Cyrot, "Gradient and hamiltonian dynamics under sampling," in 11th IFAC Symposium on Nonlinear Control Systems (NOLCOS 2019), September 2019.

[6] V. Talasila, J. Clemente-Gallardo, and A. Van der Schaft, "Discrete port-Hamiltonian systems," Systems \& Control Letters, vol. 55, no. 6, pp. 478-486, 2006.

[7] L. G. Sümer and Y. Yalçın, "A direct discrete-time IDA-PBC design method for a class of underactuated hamiltonian systems," in IFAC World Congress, vol. 18, 2011, pp. 13456-13461.

[8] S. Aoues, M. Di Loreto, D. Eberard, and W. Marquis-Favre, "Hamiltonian systems discrete-time approximation: Losslessness, passivity and composability," Systems \& Control Letters, vol. 110, pp. 9-14, 2017.

[9] S. Stramigioli, C. Secchi, A. J. van der Schaft, and C. Fantuzzi, "Sampled data systems passivity and discrete port-Hamiltonian systems," IEEE Transactions on Robotics, vol. 21, no. 4, pp. 574-587, 2005.

[10] M. Seslija, J. M. Scherpen, and A. Van Der Schaft, "Explicit simplicial discretization of distributed-parameter port-Hamiltonian systems," Automatica, vol. 50, no. 2, pp. 369-377, 2014.

[11] D. S. Laila and A. Astolfi, "Discrete-time IDA-PBC design for underactuated Hamiltonian control systems," in American Control Conference. IEEE, 2006.

[12] F. Tiefensee, S. Monaco, and D. Normand-Cyrot, "IDA-PBC under sampling for port-controlled Hamiltonian systems," in American Control Conference, 2010.

[13] S. Monaco and D. Normand-Cyrot, "Discrete-time state representations, a new paradigm," in Perspectives in Control. Springer, 1998, pp. 191-203.

[14] S. Monaco, D. Normand-Cyrot, and F. Tiefensee, "From passivity under sampling to a new discrete-time passivity concept," in 47th IEEE Conference on Decision and Control. IEEE, 2008, pp. 3157-3162.

[15] M. Mattioni, S. Monaco, and D. Normand-Cyrot, "Feedforwarding under sampling," IEEE Transactions on Automatic Control, vol. 64, no. $11,2019$.

[16] S. Monaco and D. Normand-Cyrot, "Nonlinear average passivity and stabilizing controllers in discrete time," Systems \& Control Letters, vol. 60, no. 6, pp. 431-439, 2011.

[17] A. Pavlov and L. Marconi, "Incremental passivity and output regulation," Systems \& Control Letters, vol. 57, no. 5, pp. 400-409, 2008.

[18] T. Itoh and K. Abe, "Hamiltonian-conserving discrete canonical equations based on variational difference quotients," Journal of Computational Physics, vol. 76, no. 1, pp. 85-102, 1988.

[19] M. Mattioni, S. Monaco, and D. Normand-Cyrot, "Lyapunov stabilization of discrete-time feedforward dynamics," in 56th Annual Conference on Decision and Control. IEEE, 2017, pp. 4272-4277.

[20] —, "Forwarding stabilization in discrete time," Automatica, vol. 109, p. 108532, 2019. [Online]. Available: http://www.sciencedirect.com/science/article/pii/S0005109819303930

[21] Y. Yalçin, L. G. Sümer, and S. Kurtulan, "Discrete-time modeling of Hamiltonian systems," Turkish Journal of Electrical Engineering \& Computer Sciences, vol. 23, no. 1, pp. 149-170, 2015. 